\title{
METRIZATION OF THE ONE-POINT COMPACTIFICATION
}

\author{
MARK MANDELKERN
}

(Communicated by James E. West)

\begin{abstract}
A new, more widely applicable, constructive definition of locally compact metric space is given, and a metric one-point compactification is constructed. Classically, this provides a simpler, more direct, construction of a metric on the one-point compactification of a separable locally compact metric space.
\end{abstract}

\section{INTRODUCTION}

In the constructive theory of metric spaces as developed by Errett Bishop [1], the concept of locally compact space is unique. Virtually all other metric space concepts were successfully constructivized by a judicious choice of definition from among a variety of classically equivalent conditions. In this case, however, the definition differed from the classical definition. Bishop's locally compact spaces are those in which every bounded subset is contained in a compact subset. This allows the construction of a one-point compactification, and includes many traditional locally compact spaces, such as the Euclidean spaces. However, other spaces, such as open spheres in Euclidean space, are included only if given a new metric. Here a new definition is given which resolves this difficulty.

A locally compact space should have a one-point compactification. In Bishop's constructive development of analysis, metric spaces are most useful, so this one-point compactification should be metrizable. Under the traditional definition, which merely requires that every point have a compact neighborhood, there are locally compact metric spaces whose one-point compactifications are not metrizable; thus this definition cannot be used in a constructive development. Metrizability of the one-point compactification requires that the point at infinity be the intersection of countably many neighborhoods, so the space must be $\sigma$-compact. Thus $\sigma$-compactness is used here for the definition of locally compact space, with the addition of a strong monotonicity condition on the sequence of compact subsets. The condition ensures that each point has a compact neighborhood, and allows the construction of a metric one-point

Received by the editors July 25, 1988 and, in revised form, January 3, 1989.

1980 Mathematics Subject Classification (1985 Revision). Primary 54E45; Secondary 54E35.

Key words and phrases. Locally compact, one-point compactification, metric, constructive. 
compactification. Classically, for locally compact metric spaces, the definition is equivalent to separability. Appendix A in [1] discusses the appropriateness of the metrizability and separability restrictions, from the constructive point of view.

The property of local compactness used here is hereditary for certain open subsets, and invariant under certain continuous mappings. Among the locally compact spaces will be the Euclidean spaces, open spheres in Euclidean space, metric complements of located sets in locally compact spaces, and spaces satisfying Bishop's constructive definition, all with no change of metric. Finally, the definition is characterizing; any metric space which has a metric one-point compactification is locally compact.

These results constitute a constructivization of the classical theorem which characterizes locally compact spaces $X$ with metrizable one-point compactifications $\hat{X}$ as those having a countable base [4; XI, 8.6]. While the classical proof uses standard metrization methods, embedding $X$ in a product of intervals, here we give a simple and direct definition of a metric for $\hat{X}$, based on a given metric for $X$.

\section{LOCALLY COMPACT SPACES}

A metric space $(X, \rho)$ is compact if it is totally bounded and complete. A subset $F$ of a metric space $X$ is located if the distance $\rho(x, F)$ to the subset may be measured from any point $x$ in $X$. Compact subsets are always located. The metric complement $X-F$ of a located set $F$ is the set of points situated at a positive distance from $F$. If $X$ is compact, and $g: X \rightarrow \mathbf{R}$ is uniformly continuous, then the set $\{x \in X: g(x) \leq r\}$ is void or compact for all but countably many real numbers $r$. These and other constructive properties of metric spaces are developed in [1] or [2].

For any set $F$ in a metric space $X$, and any $r>0$, we write $F(r) \equiv\{x \in$ $X: \rho(x, y)<r$ for some $y \in F\}$. When $F$ is located, then $F(r)=\{x \in$ $X: \rho(x, F)<r\}$. If $F(r) \subset G$ for some $r>0$, we will say that $G$ is a uniform neighborhood of $F$. Classically, any neighborhood of a compact set is a uniform neighborhood.

Definitions. A metric space $X$ is locally compact if it is a countable union $\bigcup_{k=1}^{\infty} H_{k}$ of compact subsets, where each set $H_{k+1}$ is a uniform neighborhood of $H_{k}$.

A bijection $\varphi: X \rightarrow Y$ between locally compact metric spaces is a homeomorphism if both $\varphi$ and its inverse $\varphi^{\leftarrow}$ are uniformly continuous on compact subsets.

Theorem 1. The following metric spaces are locally compact:

(a) Any compact space.

(b) The Euclidean spaces $\mathbf{R}^{n}$.

(c) Open spheres in $\mathbf{R}^{n}$.

(d) Any nonvoid metric complement of a located set in a locally compact space. 
(e) Any nonvoid space in which any bounded subset is contained in a compact subset.

Proof. It suffices to prove (d) and (e). Let $X=Y-F$ be the nonvoid metric complement of the located set $F$ in the locally compact space $Y=\bigcup_{k=1}^{\infty} H_{k}$, where each set $H_{k}$ is compact, $H_{k}\left(c_{k}\right) \subset H_{k+1}$, and $\left\{c_{k}\right\}$ decreases and tends to zero. Construct a decreasing sequence $\left\{d_{k}\right\}$ so that $0<d_{k}<c_{k}$ and $G_{k} \equiv\{x \in$ $\left.H_{k}: \rho(x, F) \geq d_{k}\right\}$ is compact. Then $X=\bigcup_{k=1}^{\infty} G_{k}$, and $G_{k}\left(d_{k}-d_{k+1}\right) \subset G_{k+1}$ for all $k$.

Under the condition of (e), fix a point $x_{0} \in X$. If suitable compact sets $H_{i}$ have been constructed for all $i<k$, construct a sphere of radius $\geq k$ about $x_{0}$ which contains $H_{k-1}(1)$, and construct a compact set $H_{k}$ containing this sphere.

\section{THE ONE-POINT COMPACTIFICATION}

Definitions. Let $X$ be a locally compact space. Adjoin a point $x_{1}$ to $X$, obtaining a set $X_{1}$ with $X$ as a subset. We will construct a real-valued function $h$ on $X$, and will then define

$$
\begin{aligned}
d(x, y) & \equiv \rho(x, y) \wedge(h(x)+h(y)), \quad(x, y \in X) \\
d\left(x, x_{1}\right) & \equiv h(x), \quad(x \in X) \\
d\left(x_{1}, x_{1}\right) & \equiv 0 .
\end{aligned}
$$

It will be shown that $d$ is a metric on $X_{1}$, under which $X_{1}$ is totally bounded. The completion $(\hat{X}, \hat{\rho})$ of $\left(X_{1}, d\right)$ will be called the one-point compactification of $(X, \rho)$. The mapping $i: X \rightarrow \hat{X}$ is the restriction to $X$ of the usual inclusion map $i_{1}: X_{1} \rightarrow \hat{X}$ of a space into its completion. The image of $x_{1}$ in $\hat{X}$ is denoted $\infty$ and called the point at infinity.

Theorem 2. Every locally compact metric space $(X, \rho)$ has a metric one-point compactification $(\hat{X}, \hat{\rho})$. The mapping $i: X \rightarrow \hat{X}$ is a uniformly continuous injection, and a homeomorphism between $X$ and $\hat{X}-\{\infty\}$.

Proof. For each $k$, construct $c_{k}>0$ so that $H_{k}\left(c_{k}\right) \subset H_{k+1}$ and $\left\{c_{k}\right\}$ decreases and tends to zero. Define

$$
h(x) \equiv \bigvee_{k=1}^{\infty}\left(c_{k}-\rho\left(x, H_{k}\right)\right), \quad(x \in X) .
$$

For any given point $x$, the indicated supremum is equal to the maximum of finitely many terms. Thus $h$ is well-defined, with $0<h \leq c_{1}$. It is clear that

$$
\begin{array}{ll}
h(x) \geq c_{k} & \text { when } x \in H_{k}, \\
h(x)<c_{k} & \text { when } x \in X-H_{k} .
\end{array}
$$

We now show that

$$
|h(x)-h(y)| \leq \rho(x, y), \quad(x, y \in X) .
$$


Given any $\varepsilon>0$, choose an integer $k$ such that $c_{k}-\rho\left(x, H_{k}\right)>h(x)-\varepsilon$. Then $c_{k}-\rho\left(y, H_{k}\right) \leq h(y)$, and it follows that $h(x)-h(y)<c_{k}-\rho\left(x, H_{k}\right)+$ $\varepsilon-c_{k}+\rho\left(y, H_{k}\right) \leq \rho(x, y)+\varepsilon$.

Define the function $d$ as in (1). To show that $d$ is a metric on $X_{1}$, first note that if $x, y \in X_{1}$ and $x \neq y$, then $d(x, y)>0$. Now let $x, y, z \in X_{1}$. To show that $d(x, y) \leq d(x, z)+d(z, y)$, first consider the case in which exactly one of these three points is $x_{1}$. If $x=x_{1}$, then the triangle inequality reads $h(y) \leq h(z)+d(z, y)$, or $h(y)-h(z) \leq d(z, y)$. Since $h(y)-h(z) \leq \rho(z, y)$ by (4), and also $h(y)-h(z) \leq h(y)+h(z)$, this follows from (1). The case $y=x_{1}$ is similar. If $z=x_{1}$, then $d(x, y) \leq h(x)+h(y)=d(x, z)+d(z, y)$. Now let $x, y, z \in X$, and let $\varepsilon>0$. Either (1) $\rho(x, z)<d(x, z)+\varepsilon / 2$ or (2) $h(x)+h(z)<d(x, z)+\varepsilon / 2$. Similarly, either (a) $\rho(z, y)<d(z, y)+\varepsilon / 2$ or (b) $h(z)+h(y)<d(z, y)+\varepsilon / 2$. In case (1a), we have $d(x, y) \leq \rho(x, y) \leq$ $\rho(x, z)+\rho(z, y)<d(x, z)+d(z, y)+\varepsilon$. In case (1b) it follows from (4) that $d(x, y) \leq h(x)+h(y) \leq \rho(x, z)+h(z)+h(y)<d(x, z)+d(z, y)+\varepsilon$, and case $(2 \mathrm{a})$ is similar. Finally, in case $(2 \mathrm{~b}), d(x, y) \leq h(x)+h(y)<d(x, z)+$ $d(z, y)+\varepsilon$.

To show that $X_{1}$ is totally bounded, let $\varepsilon>0$ and choose $k$ so that $c_{k}<\varepsilon$. Construct a finite $\varepsilon$ approximation $A$ to $H_{k+1}$ and define $B \equiv A \cup\left\{x_{1}\right\}$. For any point $x \in X$, either $x \in H_{k+1}$ or $x \in X-H_{k}$. In the first case, there is a point $a \in A$ such that $\rho(x, a)<\varepsilon$. In the second case, $d\left(x, x_{1}\right)=h(x)<$ $c_{k}<\varepsilon$. It follows that $B$ is a finite $\varepsilon$ approximation to $\left(X_{1}, d\right)$.

Thus $\hat{X}$ is compact. Since $h>0$ on $X$, the mapping $i$ is an injection. Since $d \leq \rho$, the mapping $i$ is uniformly continuous. It follows from (1) that

$$
\hat{\rho}(i(x), \infty)=h(x), \quad(x \in X) .
$$

To show that $i(X)=\hat{X}-\{\infty\}$, first note that one inclusion follows from (5). Now let $y \equiv\left\{y_{n}\right\}$ be a point of $\hat{X}$ with $\hat{\rho}(y, \infty)>0$, and choose $k$ so that $\hat{\rho}(y, \infty)>c_{k}$. Then $\hat{\rho}\left(i_{1}\left(y_{n}\right), \infty\right)>c_{k}$ eventually. Thus we may assume that $\left\{y_{n}\right\}$ lies in $X$, so $h\left(y_{n}\right)>c_{k}$ and $y_{n} \in H_{k}$ for all $n$. Since $\left\{y_{n}\right\}$ is $d$-Cauchy, and $h$ is bounded away from zero on $\left\{y_{n}\right\}$, it follows from (1) that $\left\{y_{n}\right\}$ is also $\rho$-Cauchy. Thus there is a point $x$ in $H_{k}$ such that $y_{n} \rightarrow x$ with respect to $\rho$, and it follows that $y=\lim i\left(y_{n}\right)=i(x)$.

Now let $K$ be a compact subset of $i(X)$. Using [5, Lemma 5.3], construct a point $y$ in $K$ such that $\hat{\rho}(\infty, y) \leq 2 \hat{\rho}(\infty, K)$. Since $y \in \hat{X}-\{\infty\}$, this, together with (5), shows that $h$ is bounded below on $i^{\leftarrow}(K)$. Hence $i^{\leftarrow}$ is uniformly continuous on $K$.

Remarks. (i) The definition of $h$ may be illustrated by the example in which $H_{k} \equiv[0, k]$ and $c_{k} \equiv 1 / k$.

(ii) If $X$ is not compact and, moreover, each metric complement $X-H_{k}$ is nonvoid, then $X$ is dense in $\hat{X}$. If $X$ is compact and, moreover, $X=H_{k}$ for some $k$, then $\infty$ is an isolated point of $\hat{X}$.

(iii) For any compact subset $K$ of $X$, the following are equivalent: 
(a) $K \subset H_{k}$ for some integer $k$.

(b) $\hat{\rho}(\infty, i(K))>0$. compact.

(c) The image $i(K)$ under the (uniformly continuous) mapping $i$ is

(d) The (positive, uniformly continuous) function $h$ has a positive infimum on $K$.

For a compact subset $K$ of the open disk, the function $t \rightarrow \rho\left(e^{2 \pi i t}, K\right)$ relates these conditions with the question of whether a positive, uniformly continuous function on the closed interval has a positive infimum. This is a fundamental, constructively indeterminate, problem; see [1, p. 151] and [3, Ch. 6].

\section{INVARIANCE AND CHARACTERIZATION}

We state the invariance theorem in a form general enough to include the canonical mapping of the plane onto an open disk.

Theorem 3. Let $X$ be a locally compact metric space, and let the mapping $\varphi: X \rightarrow Y$ be a bijection onto a metric space $Y$, such that $\varphi$ is uniformly continuous on compact subsets of $X$, and $\varphi^{\leftarrow}$ is uniformly continuous on some uniform neighborhood of $\varphi(K)$, for each compact subset $K$ of $X$. Then $Y$ is locally compact.

Proof. Let $X=\bigcup_{k} H_{k}$, where each $H_{k+1}$ is a compact uniform neighborhood of $H_{k}$. Define $G_{k} \equiv \varphi\left(H_{k}\right)$ for all $k$; thus $Y=\bigcup_{k} G_{k}$. Since $H_{k}$ is compact, $\varphi$ is a metric equivalence on $H_{k}$; thus $G_{k}$ is compact. If $H_{k}(\varepsilon) \subset H_{k+1}$, and $\delta \equiv \omega(\varepsilon) \wedge r$, where $\omega$ is a modulus of continuity for $\varphi^{\leftarrow}$ on $G_{k}(r)$, then $G_{k}(\delta) \subset G_{k+1}$.

Corollary. Any metric space having a metric one-point compactification with the properties in Theorem 2 is locally compact.

\section{REFERENCES}

1. E. Bishop, Foundations of constructive analysis, McGraw-Hill, New York, 1967.

2. E. Bishop and D. Bridges, Constructive analysis, Springer, Berlin, 1985.

3. D. Bridges and F. Richman, Varieties of constructive mathematics, Cambridge University Press, Cambridge, 1987.

4. J. Dugundji, Topology, Allyn and Bacon, Boston, 1966.

5. M. Mandelkern, Constructive continuity, Memoirs Amer. Math. Soc. 277 (1983).

Department of Mathematics, New Mexico State University, Las Cruces, New Mexico 88003 